\title{
SARS-CoV-2 and acute diverticulitis: The expanding gastrointestinal manifestations of COVID-19 infection
}

\author{
Simcha Weissman ${ }^{1}$, Anna Belyayeva ${ }^{1}$, Sachit Sharma ${ }^{2}$, Muhammad Aziz², Sameh \\ Elias $^{1}$, James H. Tabibian ${ }^{3}$ \\ 'Department of Medicine, Hackensack Meridian Health, Palisades Medical Center, North Bergen, NJ, USA; \\ 2Department of Medicine, University of Toledo Medical Center, Toledo, Ohio, USA; \\ ${ }^{3}$ Division of Gastroenterology, Department of Medicine, Olive View-UCLA Medical Center, Sylmar, CA, USA
}

\section{TO THE EDITOR}

SARS-CoV-2, the novel coronavirus which emerged in late 2019, has spread rapidly and resulted in a global pandemic. Typical presenting symptoms of SARS-CoV-2 infection, that is, COVID-19, include fever, myalgias, fatigue, dry cough, dyspnea, and anosmia, with pneumonia being the most common disease complication. Although less frequent, gastrointestinal symptoms, including nausea, vomiting, abdominal pain, and diarrhea, have been reported and have in fact implicated the gastrointestinal tract as a primary system involved in the clinical expression of COVID-19. We describe the case of a patient who presented with gastrointestinal symptoms, tested COVID-19 positive, and was subsequently found to have pneumonia as well as acute diverticulitis. This report showcases the need to recognize the expanding gastrointestinal manifestations of the COVID-19 infection and remain astute to its subsequent, often nuanced, clinical implications.

A 61-year old female with a history of lupus nephritis status-post renal transplant, chronic pelvic venous thrombosis statuspost vascular stenting, hypertension, and diverticulosis presented to the emergency department (ED) with three days of progressive nausea, vomiting, abdominal pain, and diarrhea. Physical examination revealed tachycardia, tachypnea, bilateral rales, and tenderness in the left lower abdomen. Laboratory tests revealed a leukocyte count of $8.3 \times 103 / \mu \mathrm{L}$, a neutrophil-to-lymphocyte ratio of 6.65 , erythrocyte sedimentation rate of 127 $\mathrm{mm} / \mathrm{h}$ (normal $0-15 \mathrm{~mm} / \mathrm{h}$ ), and d-dimer of 1,251 (normal $<500 \mathrm{ng} / \mathrm{mL}$ ). SARSCoV-2 pharyngeal swab rRT-PCR was positive. Chest radiograph revealed bilateral pulmonary opacities and consolidation. As the patient complained of continued abdominal pain, computed tomography (CT) abdomen and pelvis was ordered and revealed sigmoid colonic diverticular wall thickening and adjacent pericolonic fat stranding, consistent with acute diverticulitis. With bowel rest, intravenous antibiotics, analgesics, and supplemental oxygen, the patient improved and was discharged home after four days.

To our knowledge, while recent case reports have described gastrointestinal symptomatology of COVID-19, this represents the first reported case of COVID-19 associated acute diverticulitis. While the pathophysiologic mechanism is unclear, as with many of the manifestations of COVID-19, we hypothesize that viral entry via colonocyte angiotensin-converting enzyme 2 (ACE-2) receptors or a systemic inflammatory reaction may have been responsible. ${ }^{[1]}$ Viral strain may also impact differential manifestations of COVID-19. ${ }^{[1]}$ Interestingly, on a similar note, the etiopathogenesis of acute diverticulitis also remains uncertain, but the role of viral infection in a subset of cases has been postulated. ${ }^{[2,3]}$ Notably, as the gastrointestinal symptomatology in this case could have been attributed solely to viral illness, imaging to make a diagnosis of acute diverticulitis may never have been 
pursued, and in fact was initially deferred. Awareness of this manifestation can help prevent overseeing common and acute gastrointestinal pathology of COVID-19. Moreover, gastrointestinal symptomatology are of unique significance in COVID-19 patients, in contrast to other viral illnesses, owning to the fact that they often appear early and worsen during the disease course. ${ }^{[4]}$

Thus, while COVID-19-induced diverticulitis remains seemingly rare and largely unexplored, clinicians should maintain a broad differential diagnosis in COVID positive patients presenting with gastrointestinal symptoms. ${ }^{[4]}$ Additionally, on this basis, we believe it is vital to institute SARS-CoV-2 precautions in patients who present with either respiratory or gastrointestinal symptoms amidst the current pandemic. Moreover, as many aspects of this disease continue to be appreciated, clinicians should not shy away from ordering abdominal imaging when other/concomitant pathology is suspected in order to help facilitate earlier and potentially impactful real-time diagnosis and treatment.

\section{Conflict of Interests}

The authors declare to have no competing interests.

\section{REFERENCES}

1. Patel KP, Patel PA, Vunnam RR, Hewlett AT, Jain R, Jing R, et al. Gastrointestinal, hepatobiliary, and pancreatic manifestations of COVID-19. J Clin Virol 2020; 128: 104386.

2. Schieffer KM, Kline BP, Harris LR, Deiling S, Koltun WA, Youchum GS. A Differential Host Response to Viral Infection Defines a Subset of Earlier-Onset Diverticulitis Patients. J Gastrointestin Liver Dis 2018; 27: 249-55.

3. Hollink N, Dzabic M, Wolmer N, Bostrom L, Rahbar A. High Prevalence of an Active Human Cytomegalovirus Infection in Patients with Colonic Diverticulitis. J Clin Virol 2007; 40: 116-9.

4. Galanopoulos M, Gkeros F, Doukatas A, Karianakis G, Pontas C, Tsoukalas N, et al. COVID-19 pandemic: Pathophysiology and manifestations from the gastrointestinal tract. World J Gastroenterol 2020; 26: 4579-4588.

How to cite this article: Weissman S, Belyayeva A, Sharma S, Aziz M, Elias S, Tabibian JH. SARS-CoV-2 and acute diverticulitis: The expanding gastrointestinal manifestations of COVID-19 infection. J Transl Intern Med 2021; 9: 59-60. 